\title{
RESEARCH ON THE PROTECTION OF HAIYAN HALL EARTHEN STEREOBATE RELIC SITE AT YUANMINGYUAN PARK
}

\author{
Lin Yanqiu, Xiao Jinliang, Qin Hai \\ TSINGHUA HERITAGE INSTITUTION FOR DIGITIZATION. \\ D004, District No. 41 Shangdi West Road, Haidian, Beijing, China . \\ linyanqiu@thid.cn, xiaojinliang@thid.cn, qinhai@thid.cn,
}

KEY WORDS: Haiyan Hall at Yuanmingyuan Park, Earthen Relic Site, history, protection.

\begin{abstract}
:
The Haiyan Hall Earthen Relic Site at Yuanmingyuan Park is an earthen relic with significant historical value and special structure stress. Starting with the historical research of Haiyan Hall Earthen Relic Site at Yuanmingyuan Ruins, this paper offers an in-depth knowledge of the original site construction craft and proposes a protection scheme aiming at addressing the current problems at the relic site and achieving its continuity and readability by combining modern technology and abiding by the principles of relics protection.
\end{abstract}

Yuanmingyuan ruins, located in the east of San-Shan-Wu-Yuan Area, Haidian district of Beijing, China, is one of the famous imperial garden in Qing Dynasty and currently a Chinese national archaeological site park.

Situated at the northeast of Yuanmingyuan Park, European Palace scenic, built in 1759, consists of more than ten westernstyle buildings and courtyards. As an important part of cultural exchange, it marks the first introduction of European gardening art into Chinese imperial gardens and possesses a crucial position in the landscape architecture history of China and the whole world. However, in 1860 it was destroyed by British and French troops and suffered four catastrophes in the following 150 years. For this reason, the remaining sites and relics of the European Palaces cannot reach $20 \%$ of its original building.

Haiyan Hall, located in the European Palaces scenic, is the largest landscape architecture of the original scenic spot. In front of it, there is the famous Zodiac Fountain.

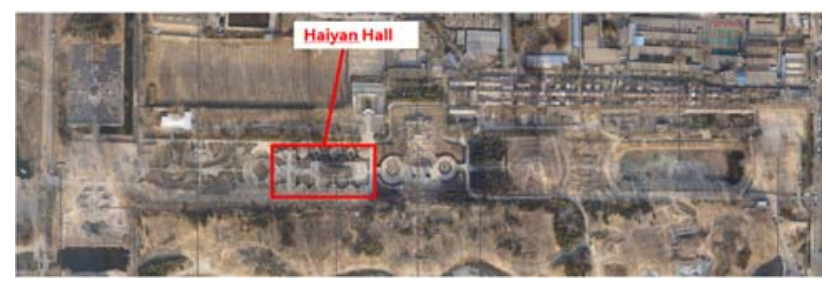

Figure 1.Location Map of Haiyan Hall

Now, Haiyan Hall relic site only left some stone parts and one earthen stereobate, which is the largest building structure in the remnants of Yuanmingyuan Park. Having been suffering from severe stress from itself, natural and man-made destruction, it calls for human intervention to achieve the protection purpose. Nevertheless, in the face of such a relic, instead of simply resorting to conventional techniques, we should not only look into its past, but also apply modern scientific and technological means in protection programs.

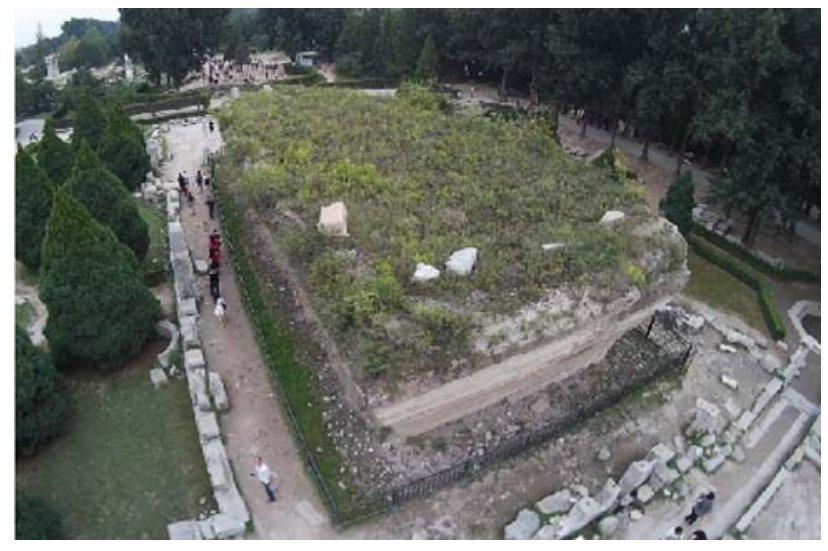

Figure 2 . The present situation of the earthen relic site

\section{THE OVERVIEW OF HISTORY}

\subsection{The Historical Architectural Forms of Haiyan Hall}

The current earthen relic site of Haiyan Hall had not formed after the destruction in the 19th century. However, when it was damaged, its rough shape was retained, especially the Water Storage Building, where the earthen relic is located, reserving comparatively complete architectural form.

Based on the pictures of each period and available drawing files, copperplate etchings and literatures, and combined with the actual measurement of the site by 3D laser scanning technology, it can be inferred that the Haiyan Hall and its attached landscape covers an area of about 8,100 $\mathrm{m}^{2}$. From west to east, it is composed of the main building, corridor, West Water Carriage House, Xi Sea, East Water Carriage House, and Eastern Steps. The overall architectural style is baroque while the detail sections is merged with traditional Chinese elements and Chinese-style hip roof is pervasive in the building. The appearance of the western and eastern ends of the $\mathrm{H}$-shaped Water Storage Building presents as a two-tiered Water Carriage House and the middle platform a reservoir, commonly known as Xi Sea. The current earthen relic site is at the bottom of the 
Xi Sea. The preliminary archaeological study proves that it shall be the bedding part of the sea.

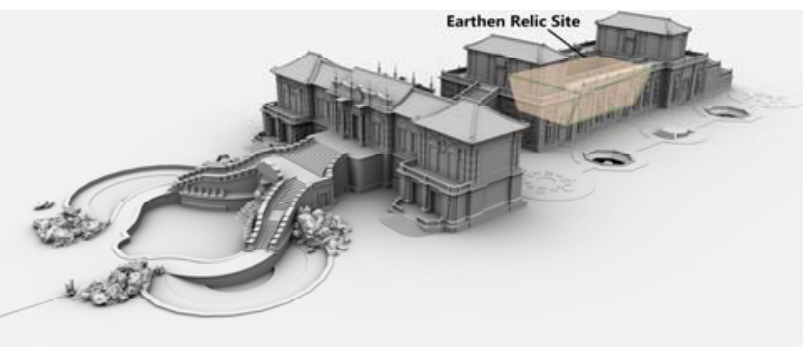

Figure 3.The Restored Map of Haiyan Hall

\subsection{The construction practice and technology in history for the earthen relic site}

It is obvious that the now earthen relic site is only a small part of Haiyan Hall. Seen from sea historical photos, the surroundings of the earthen relic site were originally enclosed by packaged bricks which were dismantled by people gradually, causing the special form that the outward extending rammed earth in the upper part had no vertical retainer. The two northsouth arched gates at the western and eastern ends of the first floor of the Water Storage Building are true doorways while the other arched gates and windows are blank, whose inner parts are enveloped by bricks. For the external walls, the arched structure, gate post, pilaster plinth, and window arched stone are stone components while the other parts like pilasters and filler walls between pilasters are built with bricks and plastered surface.

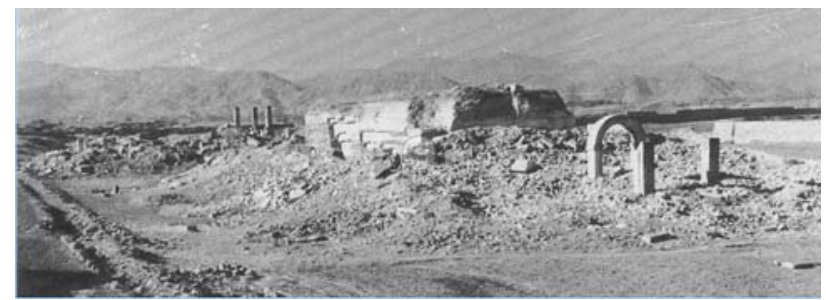

Figure4. Exposed site of rammed earth station after the block collapse at 1940 s

The earthen stereobate was built simultaneously with the external enclosed bricks in a hierarchical way, namely constructing a length of bricks while punning a layer of soil. The wall body used the same type of bricks with the size of $420 * 210 * 85 \mathrm{~mm}$ and was built in the form of alternating three stretchers with one header.

According to laboratory testing, the compressive strength of such a type of bricks is $7.08 \mathrm{MPa}$, the flexural strength $1.19 \mathrm{MPa}$, and the splitting tensile strength $1.07 \mathrm{MPa}$. Mortar clay accounts for about 55 to $80 \%$, gray material about $20 \%$, and sand about 0 to $25 \%$, and the proportion of organic matter is roughly about $15 \%$.

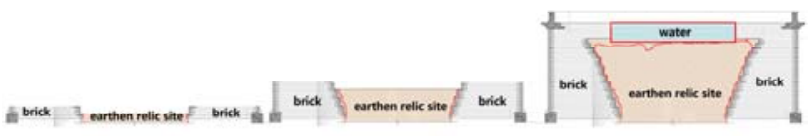

Figure 5.The stretch map for construction with rammed earth

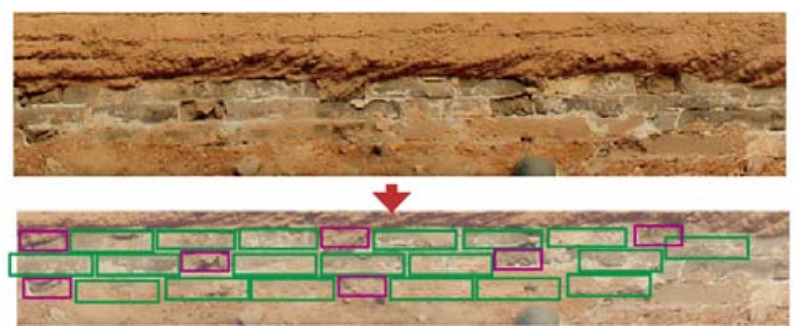

Figure 6.The stretch map for construction with bricks

\section{CURRENT SITUATION AND PROBLEMS OF THE RELIC SITE}

\subsection{Current Situation of the Relic Site}

The earthen relic site is now in the open air. As a result, the site has no other protective measures except the guard bar positioned $1.5 \mathrm{~m}$ away from the site in the $90 \mathrm{~s}$ for daily monitoring to avoid visitors' touch.

The external appearance of the earthen stereobate looks like an inverted terahedral pyramid. The residual is about $6.3 \mathrm{~m}$ high and the length of the top is $27 \mathrm{~m}$. Nine layers can be obviously seen from the outside with dense soil texture, presenting the formal feature of building with layers of rammed earth. The lines on bricks and even blue bricks coated on the earthen relic site can be clearly observed from the root and the parts of the site. There are a large number of long horizontal and vertical cracks on the body and lush plants growing at the top of the earthen stereobate.

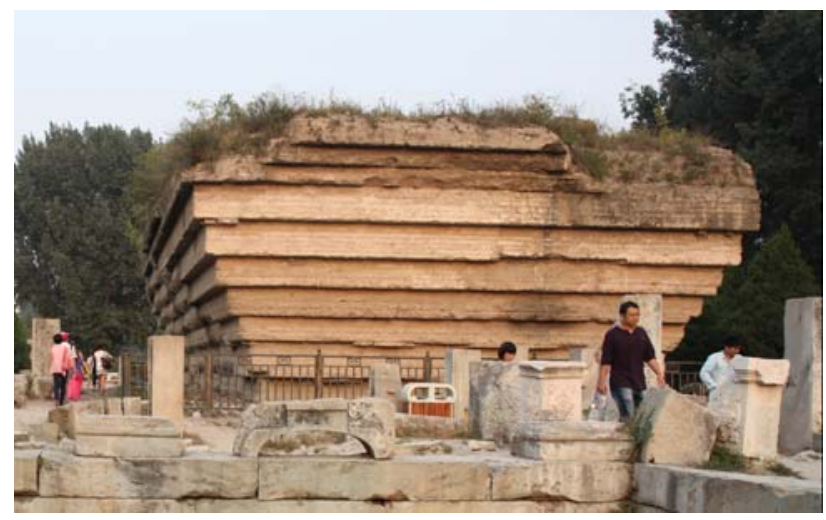

Figure 7 The present situation of the earthen relic site

\subsection{Problems of the relic site body}

Owing to exposure to sunlight and rainwater, the root system of the lush plants system on soil had resulted in loose soil body, fractures, mildew and other significant common diseases for an earthen relic site.

\subsubsection{Problems Caused by Rain Erosion}

The rain erosion problem is usually the primary problem for earthen relic sites. There are about 10 permeable holes distributing at the surrounding vertical faces of the rammed earth. All of them were watered from the top and the water gradually permeated to the surface from inside along the rammed earth where through holes are formed. Rainwater permeates along the fissures, watering, scouring and eroding the 
fissures, which will expand the relief joints and endanger the safety of the structure. Rain wash will erode the top soil layer and loose the rammed earth, giving rise to soil erosion. For that reason, the earthen relic site either at the top or around is reducing year by year. Compared with stone relics, it suffers an extremely high speed of loss and the top part of the southeast corner fell due to rain wash.

\subsubsection{Weathering and fracturing}

Direct sunlight, rain wash and plant breeding lead to soil loose, fluffy surface of the rammed earth platform, dot or piece stripping, indicating obvious weathering. Weathering at the base manifests as the rammed earth layer performing corrosion from inward.

The salt in the inner soil body will gather on the surface of the earthen relic site. Due to changes in volume caused by salt crystallization and dissolution, soil structure continues to loose for repeated expansion and contraction actions. Finally, under outer stresses, the surface of the site caves in constantly.

The long-standing problems caused by natural climate and erosion permeate from the outside to the inside gradually, which will not only affect the structural safety but also damage the appearance of the earthen body.

\subsubsection{Biological holes, plants breeding and mildew spots}

Due to the loosening effect of plant breeding on the earthen stereobate, and caves expanded from holes by animals, these holes exist widely in various parts of the façade with the diameter ranging from 0.3 to $0.7 \mathrm{~cm}$. The debris left by dead fungus like lichen represents dark brown and tawny color and attached to the upside and the surrounding of crevices at the earthen stereobate. These holes and mildew spots mainly change the appearance and surface of earthen stereobate considering its short-term and single effects, but being able to gather water has contributed to further biological breeding.

These breeding plants can reduce direct sunlight and rain erosion and exert certain impact on protecting the surface of rammed earth stereobate, but its roots are bound to grow into the rammed earth layer steadily, loosing and dismembering the layer, and leaving space for water and biological activity. Rotted acidic substances will infiltrate into the inner of rammed earth following liquid water, thus corroding the inside materials, loosening the internal structure, and causing mycete breeding increasingly at the top of the rammed earth stereobate.

\subsubsection{Problems Concerning Structure Stress}

Like ordinary earthen relic sites, Haiyan Hall earthen relic site zone has the same risk of sideways sliding and the inverted terahedral pyramid threatens the security of the rammed earth structure itself. The combined effect of rainwater, plants and other elements discussed previously highlights the structural instability problem.

The structural risk takes the following forms: long cracks appearing near the corner of four facades with a prominent slit width of $2 \mathrm{~mm}$ to $6 \mathrm{~mm}$. The combined action of external factors and internal stresses will accelerate the expansion of those cracks; the enduring stress of segregation in the vertical direction of the dangling parts leads to the slippage and separation of the earthen body, forming cracks; the free face of the corner faces the most serious danger of collapse and the overhanging upper part is also likely to go collapse.

In the checking calculation of the structural safety analysis

The gravity of the sliding wedge $(\mathrm{G})>$ the force of friction of slip surface (F)

(1) Additional load at the top will increase the value of G, causing G > F; —_External Disturbance

(2) In raining and snowing days, the soil water content will go up, reducing the soil shear strength, namely the diminution of $F$, leading to " $\mathrm{G}>\mathrm{F}$ "; _ _ Natural factors

(3) Fracture extension breaks the internal balance of soil, causing the reduction of the shear strength, namely the diminution of F, leading to " $\mathrm{G}>\mathrm{F}$ "; —_ Internal Factors

(4) The rammed earth binder (such as egg white etc.) ages over time and the bond strength decreases, namely the diminution of F, leading to " $\mathrm{G}>\mathrm{F}$ ”. _ Internal Factors -

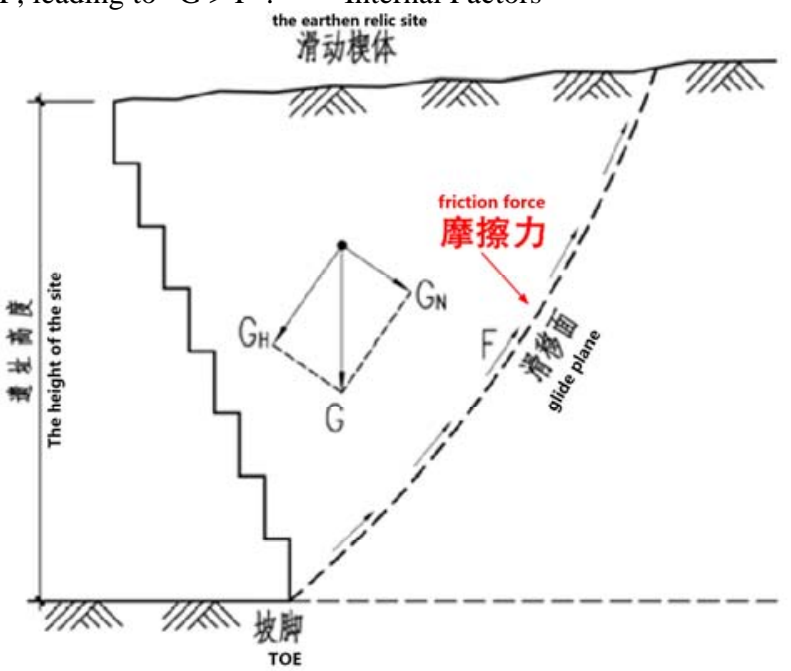

Figure 8.The analysis chart of structural stress

According to the analysis of stress field, the risk of slippage and damage exists in all sides of the rammed earth; for the position of four corners, the superimposed effect of stress will cause slippage and damage to corners because of the bidirectional slip plane, which is consistent with the present situation of rammed earth that fissures are concentrated on the corner position. Although, the upper layer of the central section and the entire rammed earth stereobate bear relative small stress and represent slighter surface breakdown, it will also face the risk of slippage over a long period of time owing to the same stress-carrying pattern with the four corners. As diseases aggravate, cracks will continuously extend, causing severe collapse to the rammed earth platform. Therefore, it is imperative to carry out rescue and reinforcement measures to Haiyan Hall earthen relic site.

\subsection{Display problems of the Relic Site}

In the face of such a special earthen stereobate, without indepth research, it is difficult even for professionals to accurately describe its functions, original appearance and other information. According to incomplete statistics, more than $90 \%$ of tourists visiting the European Palaces scenic of Yuanmingyuan Park tend to scratch their heads at the earthen relic site. It is hard for them to imagine it was originally a piece 
of water supply facilities and more difficult to imagine its original appearance. Although, equipped with some illustrated pictures and audio guide service, the site cannot be displayed completely with limited explanation and indirect impressiones.

\section{PROTECTION PRINCIPLE AND KEY POINTS}

In terms of formulating protection ideas and principles, firstly, it is necessary to strictly follow international practices and its principles of firmly keeping the authenticity and integrity of relic sites in relic protection and adopt reversible engineering technology means.

Meanwhile, on account of varying degrees of damage of relic sites, implement the principle of Rescue First and only handling crisis threatening the ontological security and integrity.

Secondly, aiming at problems existing in relic site interpretation and display, relic protection is expected to make people become more familiar with its historical, cultural and engineering value.

\section{PROTECTION METHOD ANALYSIS}

Such a protection project must be a comprehensive one with multidisciplinary participation. The following parts are concentrated on analyzing the protection scheme targeted at addressing the current problems of the relic site.

\subsection{Cleaning protection}

(1) Cleaning protection is mainly for dealing with problems such as animals and plants, crystallization, and mould, etc. Conventional salt absorption is applied to draw up the crystals salt on the surface of the site. It is workable to remove the significantly raised mould and debris, whereas shaving with bamboo chips is forbidden. By virtue of identifying insect species, insect paths (if necessary) within holes by professional teams, adults and eggs are killed, and the wormholes are blocked with spodosol, one of original compositions of rammed earth.

(2) Clean up plants growing on the top of the stereobate: saw the upper branches of shrubs with junior hacksaw, leaving 20 $\mathrm{cm}$ from the root, and strip skin; cut off the leaves of weeds on the ground with spatulas. With a syringe inject Matrox360 toward the internal part of branches and grass roots and the injection quantity varies with the size of the branches and grassroots. Too much is unfavorable. A scalpel is used to cut withered and dead tree roots. Use new soil matched according to the composition ratio of the original rammed earth to fill the holes formed after plant roots decay.

(3) Block permeable holes and cracks, and remove weeds, plant debris, regolith, etc; spray water to wet the inside of cracks so that the new and old soil body bond with each other; constitute new soil in accordance with the composition ratio of the old rammed earth as blocking materials. After that, the new soil should be sifted. The particle size should be adjusted in line with the supplementary investigation condition and further refining should be done if needed. A large-scale blocking construction program can be carried out on the condition of the completion of a field test. The blocking materials are filled into the inner from the bottom to the top in batches with the thickness of each layer less than $10 \mathrm{~cm}$. After one layer of blocking materials consolidating for a period of time and no longer shrinking, next batch can be constructed. Compact the stuffed blocking materials in cracks with air compressor and prefabricated bamboo, but the compression strength should be controlled to an appropriate degree. The blocking point should be $5 \mathrm{~cm}$ higher than its surroundings.

\subsection{Reinforcement protection}

To strengthen the structure of rammed earth stereobate, multiple protection methods are taken into consideration and a more reasonable scheme is chosen following a careful comparison.

\subsubsection{Protection with carbon fiber cloth}

From the angle of mechanics analysis, as flexible material, Carbon fiber cloth can effectively balance the horizontal force produced by the destruction of soil and restrain the earthen body, but it fails to balance the vertical force produced by the destruction of soil and provide support to the soil body. So, carbon fiber reinforcement can't eliminate the hidden danger of being destroyed.

\subsubsection{Protection with holder reinforcement}

For this, horizontal steel plates are used to enclose the bottom and sides while vertical steel columns (bracing) to support and fix steel plates. At the foot of the steel column, a concrete independent foundation was built, whose own weight will produce frictional force when contact the ground, and form a steady support system. Those three parts constitute an independent stable supporting system.

With this method, the steel structure support component will not exert pressure on the earthen body of the relic site under the condition of no collapse occurring. When it collapses, the steel structure support component will be able to bear the weight of the site itself. Checking calculation shows that the size of the independent foundation plane is $3.0 \mathrm{~m} * 5.0 \mathrm{~m}$ with the height of $1.6 \mathrm{~m}$.

The characteristics of the soil on this relic site require the single block board has larger contact area against the site to ensure even stress, or the earthen strereobate will be subjected to secondary loading resulting from local stresses. Nevertheless, it is difficult to guarantee average stress between contact surfaces for the irregular shape of the earthen relic site. Steel column support features a large amount of materials and massive work amount which needs, but the foundation directly built on $t$ on the ground will bring negative influence on the landscape of the relic site.

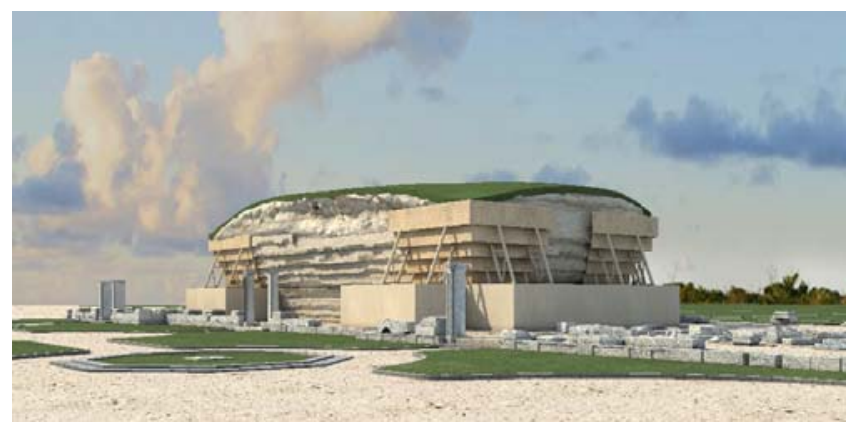

Figure 9. Effect drawing for the holder enforcement method 
4.2.3 Protecting by strengthening the brick-enclosed structure

Integrated with the original and the local structure of the earthen relic site, restoring the external structure in history according to its local forms, and adhering to the positioning principle of endowing the site with a shell having holding power, this paper proposed the brick-enclosed supporting method.

According to the original structure and material of the Water Storage Building, the brick-enclosed method was adopted to first offer support for the four corners, parts bearing weakest stress and most easily going collapse. Fill the reserved space of 20mm between brick-enclosed walls and the earthen relic site with polystyrene or the same soil with that on the relic site.

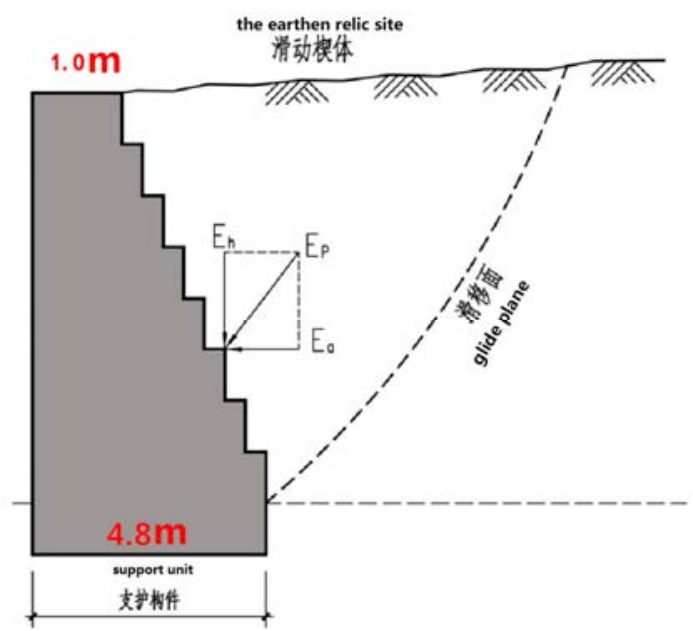

Figure 10.The analysis chart of structural stress

Judged from the residual brickwork on site, it can be inferred that the masonry material is baked bricks in cinerous. Pointing uses the white lime. Report on specific compositions has been completed. According to the actual measurement on residual building blocks, the size of a full block is $420 \mathrm{~mm}$ long, 210 $\mathrm{mm}$ wide, and $85 \mathrm{~mm}$ high and the width of the pointing is $8-10$ $\mathrm{mm}$. The existing relations between the residual brick blocks on the earthen stereobate contributes to the preliminary judgment that there was no isolation layer between them, but it is uncertain whether other bonding or water proof coatings had been used on the surface of brick blocks in masonry process and calls for further experimental analysis in the process of archaeology. The base at the south of the stereobate reserves relatively neat building blocks. It can be seen from the present situation that the method of brick masonry is alternating three stretchers with one header, constructing with staggered joints.

The calculation of the weight of the soil body and side thrust indicates that the height of the brick is $6 \mathrm{~m}$; the minimum thickness of enclosed bricks at the top is $1 \mathrm{~m}$ and that of the bottom is $4.850 \mathrm{~m}$; the self-load weight of the brick setting protection walls is around $120 \mathrm{kN} / \mathrm{m} 2$ and the total load of the bottom wall is of about $351 \mathrm{kN} / \mathrm{m}$; the position of the newlybuilt foundation is identical with that of the brick-enclosed structure in history and the quantity and the load capacity of enclosed bricks are quite larger than in history, so it can be sure that the newly-built protection walls exhibit favorable basic load capacity.
Stocky as the brick-enclosed body is, a single building block is small, making it combine with the earthen relic site flexibly and obtain even stress. It can increase or decrease the range of brick enclosed support according to changes of the relic site. As for style, enclosed bricks can be fully distinguished with the relic site, but maintain the supporting method in history, greatly enhancing the readability of the earthen relic site.

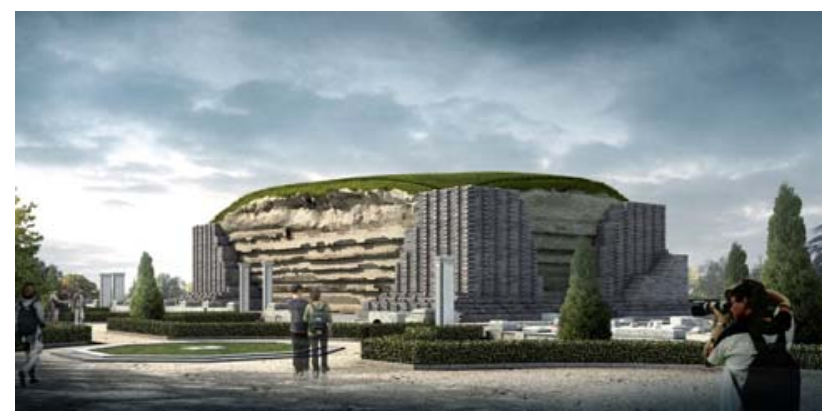

Figure11. Effect drawing for the brick-enclosed reinforcement method

\subsection{Water Proof Protection}

According to the existing status of the relic site and techniques available, commonly used waterproof ways include chemical protection, protecting by increasing waterproof layers, canopy waterproof protection.

\subsubsection{Chemical protection}

Treating the top of the earthen relic site with chemicals to achieve the purpose of waterproof has the advantage of not affecting the style of the site. However, adding chemicals will cause reaction with soil and change its properties (some chemicals have slight toxicity), making it difficult to guarantee the effectiveness and predict the negative effects of chemical materials. It is the disadvantage of chemical protection. The method may be effective in a short term, acting as a contingency measure and requiring regular chemical spray and maintenance.

\subsubsection{Canopy waterproof protection:}

Set up a canopy over the ruins to prevent the above part and sides from drifting rain water. The canopy has a transparent glass roof and a large-span truss. The overall height of the canopy is $9.9 \mathrm{~m}$, with top part $1.5 \mathrm{~m}$, the north-south span $20 \mathrm{~m}$ and the east-west length $33 \mathrm{~m}$. There are six stanchions with the diameter of $350 \mathrm{~mm}$ and the spacing of $6 \mathrm{~m}$ in the north and south sides. An independent foundation at the bottom of the column stands above the ground ruins. Its side length is $1.7 * 2.4 \mathrm{~m}$. The advantage of this method is its relatively thorough waterproof and a long-term effect. Its drawbacks include a greater influence on the style and uneven stresses owing to the independent foundation based on the ground.

\subsubsection{Waterproof layer protection:}

First, cover a layer of bentonite, 300mm in thickness, then add the waterproof layer, and spread 150 thick soil and solid soil turf. Its advantages are solving the problem of rain-proof 
relatively completely, a long-term effect, and no impact on the style. Defects are bigger load of the newly added materials at the top part, massive work amount and large disturbance on the site.

On the basis of the protection scheme for this relic site, the total thickness of solid soil layer and waterproof layer is $\mathrm{h}=0.45 \mathrm{~m}$. Generally, if the bulk density of the soil-fixation is $r=20$ $\mathrm{kN} / \mathrm{m} 3$, the pressure of soil fixation layer exerting on the relic site is $\mathrm{rh}=20 * 0.45=9 \mathrm{kN} / \mathrm{m} 2$.

The checking calculation on the stress of the edge of the site demonstrates that the added soil layer, acting as even stress, has not changed stress distribution significantly and will not affect the structural safety of the stereobate.

All of the waterproof measures discussed above encounter the same problem that the waterfall formed by free draining will erode stereobate walls and scour the ground. Hence, it is essential for roof drainage to adopt an organized form.

\section{CONCLUSION}

In the whole process of the site protection, we insist on analyzing from multiple perspectives: explore the present situation trace from the perspective of archaeological investigation, dig information from the angle of the historical research, test components from the angle of material science, and finally achieve scientific inference and implement protection methods. On the basis of a determined brickenclosed supporting and waterproof layer protection scheme, its characteristic and protection principles should be strictly grasped in the process of further design and construction to achieve the continuity and readability of the relic site.

\section{REFERENCES}

Xiao Jinliang, 2014. The "accuracy" should be added to the standard of construction site restoration. World Heritage Site 2014.10. pp 23.

He yan ,Guo Daiheng, Xiao Jingliang. Preserve Design of Historic Bridge Remains in Jiuzhou Qingyan Scenery Region of Yuanming Yuan . From the perspective of ecological civilization of urban and rural planning - 2008 China city planning annual meeting proceedings.pp1-20.

Huang Kezhong. The conservation of rock and earth structure sites [M].Beijing:China Architectuer \& Building Press,1998:4753.

Guodaiheng He Yan.Digital reconstruction Old Summer Palace. [M].Beijing: Chinese and western.2012.

Xiao Jinliang, The protection and display of large city sites -Taking the practice of Luoyang city in Sui and Tang Dynasties as an example. Architectural Journal.2012.6.pp69-73. 